University of Wollongong

Research Online

Faculty of Engineering and Information

Faculty of Engineering and Information

Sciences - Papers: Part A

Sciences

2013

Continuous manipulation and separation of particles using combined obstacle-and curvature-induced direct current dielectrophoresis

Ming Li

University of Wollongong, m1433@uowmail.edu.au

Shunbo Li

Hong Kong University of Science and Technology

Weihua Li

University of Wollongong, weihuali@uow.edu.au

Weijia Wen

Hong Kong University of Science and Technology

Gursel Alici

University of Wollongong, gursel@uow.edu.au

Follow this and additional works at: https://ro.uow.edu.au/eispapers

Part of the Engineering Commons, and the Science and Technology Studies Commons

Research Online is the open access institutional repository for the University of Wollongong. For further information contact the UOW Library: research-pubs@uow.edu.au 


\title{
Continuous manipulation and separation of particles using combined obstacle- and curvature-induced direct current dielectrophoresis
}

\author{
Abstract \\ This paper presents a novel dielectrophoresis-based microfluidic device incorporating round hurdles \\ within an S-shaped microchannel for continuous manipulation and separation of microparticles. Local \\ nonuniform electric fields are generated due to the combined effects of obstacle and curvature, which in \\ turn induce negative dielectrophoresis forces exerting on the particle that transport throughout the \\ microchannel electrokinetically. Experiments were conducted to demonstrate the controlled trajectories \\ of fix-sized (i.e. 10 or $15 \mathrm{x} \mu \mathrm{m}$ ) polystyrene particles, and size-dependent separation of 10 and $15 \mu \mathrm{m}$ \\ particles by adjusting the applied voltages at the inlet and outlets. Numerical simulations were also \\ performed to predict the particle trajectories, which showed reasonable agreement with experimentally \\ observed results. Compared to other microchannel designs that make use of either obstacle or curvature \\ individually for inhomogeneous electric fields, the developed microchannel offers advantages such as \\ improved controllability of particle motion, lower requirement of applied voltage, reduced fouling, and \\ particle adhesion, etc. (C) 2013 WILEY-VCH Verlag GmbH \& Co. KGaA, Weinheim.
}

\section{Keywords}

curvature, obstacle, combined, particles, separation, manipulation, dielectrophoresis, continuous, current, direct, induced

\section{Disciplines}

Engineering | Science and Technology Studies

\section{Publication Details}

Li, M., Li, S., Li, W., Wen, W. \& Alici, G. (2013). Continuous manipulation and separation of particles using combined obstacle-and curvature-induced direct current dielectrophoresis. Electrophoresis, 34 (7), 952-960. 


\title{
Continuous manipulation and separation of particles using combined obstacle-and curvature-induced DC dielectrophoresis
}

\author{
Ming $\mathrm{Li}^{1}$, Shunbo $\mathrm{Li}^{2}$, Weihua $\mathrm{Li}^{1}{ }^{1}$, Weijia Wen ${ }^{2}$, Gursel Alici ${ }^{1}$ \\ ${ }^{1}$ School of Mechanical, Materials and Mechatronic Engineering, University of Wollongong, \\ Wollongong, NSW, 2522, Australia \\ 2 Department of Physics, The Hong Kong University of Science and Technology, Clear Water Bay, \\ Kowloon, Hong Kong \\ Correspondence:
}

Prof. Weihua Li, School of Mechanical, Material and Mechatronic Engineering, University of Wollongong, NSW, 2522, Australia;

Prof. Weijia Wen, Depart of Physics, The Hong Kong University of Science and Technology, Clear Water Bay, Kowloon, Hong Kong.

E-mail: weihuali@uow.edu.au; phwen@ust.hk

Fax: +61-2-4221-3238; +852-2358-1652

Abbreviation: EK, electrophoretic; EO, electroosmosis; EP, electrophoresis; LOC, lab-on-a-chip; PDMS, polydimethylsiloxane; PS, polystyrene;

Keywords: Curvature, DC dielectrophoresis, Obstacle, Particle manipulation, Particle separation

Total number of words: 4755 


\section{Abstract}

This paper presents a novel dielectrophoresis (DEP)-based microfluidic device incorporating round hurdles within an S-shaped microchannel for continuous manipulation and separation of microparticles. Local nonuniform electric fields are generated due to the combined effects of obstacle and curvature, which in turn induce negative DEP forces exerting on the particle that transport throughout the microchannel electrokinetically. Experiments were conducted to demonstrate the controlled trajectories of fix-sized (i.e. 10 or $15 \mu \mathrm{m}$ ) polystyrene (PS) particles, and size-dependent separation of 10 and $15 \mu \mathrm{m}$ particles by adjusting the applied voltages at the inlet and outlets. Numerical simulations were also performed to predict the particle trajectories, which showed reasonable agreement with experimentally observed results. Compared to other microchannel designs that make use of either obstacle or curvature individually for inhomogeneous electric fields, the developed microchannel offers advantages such as improved controllability of particle motion, lower requirement of applied voltage, reduced fouling and particle adhesion, etc. 


\section{Introduction}

With the rapid development of lab-on-a-chip (LOC) devices and micro total analysis systems ( $\mu$ TAS) in recent years $[1,2]$, dielectrophoresis (DEP), the phenomenon that occurs due to a translational force exerted on a dielectric particle in a nonuniform electric field [3], has been proven as a versatile mechanism to manipulate various micro/nano scale bioparticles (i.e. DNA [4], protein [5], bacteria [6], virus [7], and mammalian cells [8]) in aqueous solution. Compared to other techniques applied in microfluidic devices, such as mechanical [9], thermal [10], magnetic [11], acoustic [12], optical [13], chemical [14] and electrical $[15,16]$ methodologies, DEP may be the most popular one due to its label-free nature, favourable scaling effects, simplicity of the instrumentation, ability to manipulate neutral bioparticles, and analysis of high selectivity and sensitivity [17-19].

Traditionally, the spatial nonuniformities required for DEP effect are generated by applying alternative current $(\mathrm{AC})$ electric fields to the microelectrodes patterned within microchannels. A variety of $2 \mathrm{D}$ or $3 \mathrm{D}$ microelectrode configurations have been developed and implemented in microfluidc devices, which have been reviewed in the literature $[19,20]$. However, such electrodebased systems suffer from fabrication complexities due to electrode construction, electrode fouling [18], and electrochemical reactions on the electrode surface [21]. These problems are not encountered in insulator-based DEP microdevices, where direct current (DC) electric fields are applied via external electrodes submerged in reservoirs to create electric field gradients around insulating objects [22]. Recently, a novel technique called contactless DEP has been developed [23, 24], in which the electrodes and the sample are separated by insulating microbarries. Although it is advantages in terms of no sample contamination and bubble generation, the fabrication process needs photolithography for sacrificial template, and subsequent Deep Reactive Ion Etching (DRIE) for master stamp, which is time-consuming, expensive and requires aggressive chemicals.

Overall, two main approaches have been successfully utilized in insulator-based DEP microdevices to generate the required nonuniform electric fields. The first one is the use of electrically insulated 
obstacles embedded in straight microchannels, including posts $[25,26]$, rectangular/triangular hurdles [27-30], ridge [31], oil droplet [32], and oil menisci [33]. However, these obstacle-based designs have such limitations as locally amplified electric fields around obstacles, large transmembrane voltages and shear stresses on cells, and induced significant Joule heating. Moreover, the device is prone to fouling due to particle clogging or adhesion [34]. Alternatively, curved insulating microchannels were employed for spatial nonuniformities, including sawtooth [35], serpentine [36], circular [37, 38], spiral [39, 40], and waved [41] ones. Although high-intensify local electric fields are avoided, this curvature-based method requires sufficiently large applied DC voltage and/or long curved section for effective performance of the device, inducing complexities in terms of operation and fabrication. In addition, the device is more sensitive to contamination (i.e. particle adhesion on channel wall), because long channel increases the possibility of surface inhomogeneity, which could disturb the electroosmotic flow for particle transport [42].

In this work, we developed a novel design coupling the effects of obstacle and curvature to generate electric field gradients required for the DEP effect, where multiple round hurdles were embedded within an S-shaped microchannel to achieve continuous particle manipulation and separation. By using hurdles together with curved channel for the spatial nonuniformities, the aforementioned adverse effects of using each approach individually (i.e. locally applied electric fields, particle clogging, and large applied voltage, etc.), have been significantly reduced. Moreover, the necessary functionality of the microfluidic device is facilitated due to the increase of approach for particle manipulation, as well as the integration of pre-focusing and post-manipulating processes. How applied voltages at inlet and outlets affect the trajectory of particle motion was studied by manipulating 10 or $15 \mu \mathrm{m}$ polystyrene (PS) particles flowing through the microchannel. Moreover, the separation functioning of the design was verified by effectively and successfully directing 10 and $15 \mu \mathrm{m}$ PS particles into distinct outlets simultaneously. Both experimental and numerical results were presented, indicating an acceptable coincidence with each other. 


\section{Materials and methods}

\subsection{Theory}

Particles suspended in an electrically conducting liquid under the influence of external electric field are subjected to electrophoretic, electroosmotic and dielectrophoretic effects. The combination of fluid electroosmosis (EO) and particle electrophoresis (EP) is termed electrokinetic (EK) flow, resulting in the electrokinetic velocity of particles written as [43]

$\mathbf{u}_{E K}=\mu_{E K} \mathbf{E}=\mathbf{u}_{E O}+\mathbf{u}_{E P}=\left(\mu_{E O}-\mu_{E P}\right) \mathbf{E}$

where $\mu_{E K}, \mu_{E O}=-\varepsilon_{m} \zeta_{w} / \eta$, and $\mu_{E P}=-\varepsilon_{m} \zeta_{p} / \eta$ are electrokinetic, electroosmotic and electrophoretic mobility, respectively. $\varepsilon_{m}$ and $\eta$ are the permittivity and dynamic viscosity of the suspending medium, respectively. $\zeta_{w}$ and $\zeta_{p}$ represent, respectively, the zeta potentials of the channel wall and particle. $\mathbf{E}$ is the electric field vector. Eq. 1 shows that the electrokinetic velocity $\left(\mathbf{u}_{E K}\right)$ of the particle is linearly proportional to the local electric field (E), leading to the particle transport along the electric lines.

Using the dipole moment method, the time-average DEP force acting on a dielectric spherical particle in a nonuniform DC electric field is given by [44]

$\mathbf{F}_{D E P}=(1 / 2) \pi \varepsilon_{m} d^{3} f_{C M}(\mathbf{E} \cdot \nabla \mathbf{E})$

and the induced dielectrophoretic velocity is expressed as

$\mathbf{u}_{D E P}=\mu_{D E P}(\mathbf{E} \cdot \nabla \mathbf{E})=\left(\varepsilon_{m} d^{2} f_{C M} / 6 \eta\right) \cdot(\mathbf{E} \cdot \nabla \mathbf{E})$

where $\mu_{D E P}$ is dielectrphoretic mobility, $d$ is the particle diameter, $f_{C M}=\left(\sigma_{p}-\sigma_{m}\right) /\left(\sigma_{p}+2 \sigma_{m}\right)$ is known as the Clausius-Mossotti (CM) factor, $\sigma_{p}$ and $\sigma_{m}$ are the electric conductivities of particle and the suspending medium, respectively. If the particle is less conductive than the suspending medium $\left(\sigma_{p}<\sigma_{m}\right), \mathrm{CM}$ factor will be negative $\left(f_{C M}<0\right)$, inducing a negative DEP force that repels particle away from the region of higher electric field.

\subsection{Microchannel fabrication and design}


The polydimethylsiloxane (PDMS) microfluidic channel was fabricated using standard softlithography technique, and a detailed fabrication process can be found in our previous work [41]. As shown in Figure 1a, the microfluidic chip is composed of two semi-circular channels that each integrating with three round hurdles from the inner wall, one inlet $(A)$ and two outlet (B and $C$ ) reservoirs, and three straight connecting microchannels. Both semi-circular channels are $300 \mu \mathrm{m}$ in width, having a large curvature of $600 \mu \mathrm{m}$ and a small curvature of $300 \mu \mathrm{m}$. The round hurdles in the first and second curved channel have radius of, respectively, $150 \mu \mathrm{m}$ and $170 \mu \mathrm{m}$, creating $40 \mu \mathrm{m}$ and $60 \mu \mathrm{m}$-wide gaps between hurdle and outer wall, respectively. All three straight connecting channels have a width of $300 \mu \mathrm{m}$ and a length of $0.5 \mathrm{~cm}$, and the microchannel is approximately 40 $\mu \mathrm{m}$ deep. All three reservoirs at the inlet and outlets are $3 \mathrm{~mm}$ in height and $6 \mathrm{~mm}$ in diameter.

The mechanism of the proposed design is illustrated schematically in Figure $1 \mathrm{~b}$, where the electric field lines (or equivalently the streamlines with black arrows indicating the direction) and contours of the electric field strength (the darker the stronger) within the microchannel are presented. It can be found that due to the combined effects of obstacle and curvature, local nonuniform electric fields are generated throughout the microchannel excluding the straight sections with uniform width. Consider a particle subjected to negative DEP effect passing though the microchannel under the electrokinetic effect (a combined effect of EOF and EP), repulsive DEP forces (represented by dark blue arrows, strongest near the hurdle edge and decay as increasing distance away from the hurdle) will be exerted on the particle all along its movement (see Figure 1c).

The proposed microchannel taking advantage of both obstacle and curvature for continuous particle manipulation and separation is composed of two units of hurdle-embedded curved channel. The first unit was designed herein to confine particles into a stream close to the hurdle edge within the second unit for subsequent switching or sorting process. Since incoming position of particles could affect its trajectory shift after passing the hurdle (refer to the previous work of Kang et al. [28]), the integration of pre-focusing and post-manipulating processes will allow improvement in the device 
performance. The round hurdle is employed to create a smoothly varied electric field due to its curved shape, which avoids greatly strong and highly nonuniform electric fields, and the accompanying adverse effects (i.e. unprecedented particle motion and biological damage) at the sharp tips of other hurdle geometries (i.e. rectangular and triangular). Moreover, the use of multiple hurdles enhances the generated DEP forces on particles and increases the controllability of the particle motion, facilitating the functionalities of the proposed design [30].

\subsection{Microparticle preparation}

In this study, we used two types of particles with varied sizes (Fluospheres, Invotrogen, CA, USA): yellow-green fluorescent $10 \mu \mathrm{m}$ and blue fluorescent $15 \mu \mathrm{m}$ polystyrene (PS) microspheres. Both particles originally suspended in $0.15 \mathrm{M} \mathrm{NaCl}$ solution were diluted by deionized (DI) water 15 times. For separation experiment, the diluted 10 and $15 \mu \mathrm{m}$ particle solutions were mixed at a volume ratio of 1:1. The particle solutions were gently vibrated prior to be introduced into the inlet reservoir using a pipette. Moreover, the outlet reservoirs were filled with the corresponding working solution, and the liquid level in each reservoir was carefully balanced before the application of DC voltages.

\subsection{Apparatus and technique}

The electric field was generated by a DC power supply (SL1OP300/200, Spellman High Voltage Electronics Corp., Hauppauge, NY), and applied via three platinum (Pt) electrodes submerged in inlet and outlet reservoirs. The motion of particle was monitored and recorded by an inverted microscope (Olympus IX71, Tokyo, Japan) equipped with a CCD camera (DP 70, Olympus, Tokyo, Japan), and a computer having Olympus DP controller image software. The camera was run in the video mode at the speed of 15 frames per second and the acquired digital images had a resolution of $680 \times 512$ pixels. All the videos and images were post-processed by MATLAB (Mathworks Inc., Natick, MA), and the particle trajectory images were obtained by superimposing consecutive images converted from videos. 


\subsection{Numerical modelling}

To predict the trajectories of particle motion throughout the developed microchannel, we used a two-dimensional (2D) numerical model that first developed by Kang et al [28], and has also validated by other researchers in various microfluidic channel structures [36, 40, 41]. By introducing a correction factor $c$ to account for the effects of particle size, particle-particle interaction, etc. on the dielectrophoretic velocity, the velocity of particle can be written as

$\mathbf{u}_{p}=\mathbf{u}_{E K}+c \mathbf{u}_{D E P}=\mu_{E K} \mathbf{E}+c \mu_{D E P}(\mathbf{E} \cdot \nabla \mathbf{E})$

The above equation was employed in a streamline function in the electrostatics module of COMSOL 4.0 (COMSOL Inc., Burlingtion, MA) to simulate the particle trajectories. The boundary conditions include the DC voltages applied at the ends of the microchannel, and the insulating condition on channel sidewall and hurdle edge. Assuming the medium of constant electric conductivity, the electric potential field is governed by Laplace equation expressed as $\nabla^{2} \varphi=0$, and the electric field is obtained by calculating the derivative of the electric potential $\mathbf{E}=-\nabla \varphi$. At the inlet, particles were assumed massless and uniformly distributed along the channel width. In the simulation, the electrokinetic mobility, $\mu_{E K}$, and dielectrophoretic mobility, $\mu_{D E P}$, were calculated by Eq. 1 and 3, respectively. The zeta potential values of PS particles and channel wall in $10 \mathrm{mM} \mathrm{NaCl}$ solution were set to be -33 and $-54 \mathrm{mV}$, respectively $[45,46]$. The dynamic viscosity, $\eta=0.9 \times 10^{-3} \mathrm{~kg} /(\mathrm{m} \cdot \mathrm{s})$, and permittivity, $\varepsilon_{m}=6.9 \times 10^{-10} \mathrm{C} /(\mathrm{v} \cdot \mathrm{m})$ for pure water at $25^{\circ} \mathrm{C}$ were also used. As the electric conductivity of polystyrene particle in DC electric field is much smaller than that of suspending medium (i.e. $10 \mathrm{mM} \mathrm{NaCl}$ solution) used in our experiments, the $\mathrm{CM}$ factor, $f_{C M}$, which depends on electrical conductivities of both the particle and the suspending medium was set to be -0.5 . The correction factor, $C$, was obtained by matching the simulated particle trajectories to the superimposed experimental results in the same conditions, which has been demonstrated to decrease with the rise in particle size and dependent on channel geometry [36, 40, 41]. 


\section{Results and discussion}

As mentioned above, electrophoretic, electroosmotic, and dielectrophoretic effect which are combined to determine particle motion depend on such parameters as electric field and particle size. Both experiments and numerical simulations were performed to study how applied voltage affects the trajectory of particle motion, and device functionality for sorting two-sized (i.e. 10 and $15 \mu \mathrm{m}$ ) PS particles.

\subsection{Continuous manipulation of particles}

\subsubsection{Effect of applied voltage at inlet}

Figure 2 shows the comparison between experimental observed (left column: snapshot; middle column: superimposed) images and numerically predicted (right column) results of $10 \mu \mathrm{m}$ particle movement under varied inlet voltages. As shown in Figure 2a, particles in the entry region were uniformly distributed and covered almost the entire channel width. In the first curved unit, particles that initially unfocused were confined in the gap regions, and forced to move closely to the hurdle region in the second curved unit (see Figure $2 \mathrm{~b}$ ). Particle trajectories at the exit region are presented in Figures $2 \mathrm{c}$ and $2 \mathrm{~d}$ : at lower inlet voltage (c: $140 \mathrm{~V}$ ), all particles were moved out of the microchannel from outlet $B$, however, particles were directed to a confined stream in outlet $C$ at higher applied voltage (d: $340 \mathrm{~V}$ ). Moreover, by comparing the width of particle stream within the outlet in the experiment (the measured widths were 145 and $74 \mu \mathrm{m}$ for inlet voltage of 140 and 340 $\mathrm{V}$, respectively), it could be found that $10 \mu \mathrm{m}$ particles obtained a better focusing performance at higher applied voltage, which is in accord with the finding of our previous work [41]. Since DEP force depends on the gradient of the square of the electric field (according to Eq.2), fix-sized particles will experience a larger repulsive DEP effect and tend to be deflected further away from the hurdle edge at a larger inlet voltage. The right column of Figure 2 represents the numerically predicted 
trajectories of $10 \mu \mathrm{m}$ particles, which showed a reasonable agreement with the experimentally observed ones when the correction factor was set as 0.5 .

\subsubsection{Effect of applied voltage at outlet}

Besides the effect of inlet voltage, we also studied experimentally and numerically how applied voltages at the outlet affect the movement of $10 \mu \mathrm{m}$ particles. Figure $3 \mathrm{a}$ and $3 \mathrm{~b}$ show particle trajectory at the exit region, when the applied voltages at outlet B were 15 and $50 \mathrm{~V}$, respectively, while the applied voltages at inlet and outlet $\mathrm{C}$ were fixed constant. In both cases, particles were observed to move in outlet $C$, however, the particle stream was close to the left-side wall (Figure 3a) and almost at channel centre region (Figure 3b) with applied voltages of 15 and $50 \mathrm{~V}$, respectively, indicating that particles could be repelled further toward the right-side wall of the outlet $\mathrm{C}$ by increasing the applied voltage at outlet B. Moreover, it was also seen that particles moved in a narrower stream at higher applied voltage (the measured stream widths were, respectively, 117 and $65 \mu \mathrm{m}$ for the voltage of 15 and $50 \mathrm{~V}$ ), indicating that the focusing performance could increase with the rise of applied voltage at outlet B.

Additionally, different trajectory shifts can also be achieved by varying applied voltage at outlet C. As shown in Figure $3 c$ and $3 d$, by increasing the outlet $C$ voltage from (c) $15 \mathrm{~V}$ to (d) $50 \mathrm{~V}, 10 \mu \mathrm{m}$ particles were not only shifted further toward the left-side channel wall of outlet B, but also focused within a tighter stream. By comparing Figure $3 a$ with Figure $3 c$, and Figure $3 b$ with Figure $3 d$, we could conclude that particles could be directed to distinct outlets by switching the applied voltages at the outlets. This is because outlet voltages could determine the flow streams at the bifurcation, hence direct the particle movement after passing the curved section, which has also been stated in the previous work of Kang et al. [29]. Moreover, larger applied voltage causes higher electric field nonuniformity, generating larger DEP forces for particle deflection in both constricted and curved regions. The numerically predicted trajectories for $10 \mu \mathrm{m}$ particles at the exit region are presented in 
the right column of Figure 3, which seem to match the experimental results closely with the correction factor fixed at 0.5 .

In similar analysis, we studied experimentally and numerically how the applied voltages at inlet and outlets affect the movement of $15 \mu \mathrm{m}$ particles. Figure 4 represents the experimentally observed (left column: snapshot; middle column: superimposed images) trajectories of $15 \mu \mathrm{m}$ particles at the exit region of the microchannel under different combinations of inlet and outlet voltages: (a) when applied voltages at inlet $A$, outlet $B$ and outlet $C$ were, respectively, 90 and 0 and $10 \mathrm{~V}$, particles moved out of the microchannel in a confined stream from outlet $B$; (b) by increasing the inlet voltage to $180 \mathrm{~V}$ but remaining outlet voltages, particles were pushed further from round hurdles and directed to outlet $\mathrm{C}$ in a narrower stream due to larger repulsive DEP force; (c) further increased the outlet $\mathrm{C}$ voltage to $30 \mathrm{~V}$, but fixed outlet $\mathrm{B}$ to be grounded, particles were diverted to move from outlet B again due to the re-distribution of the flow at the bifurcation. By comparing Figure 4a with $4 \mathrm{~b}$, and Figure $4 \mathrm{~b}$ with $4 \mathrm{C}$, it can be found that both applied voltages at inlet and outlets could affect particle trajectory, and $15 \mu \mathrm{m}$ particles can be directed into either outlet $\mathrm{B}$ or outlet $\mathrm{C}$ depending on inlet and outlet voltages. In addition, with the increase of inlet and/or outlet voltage, $15 \mu \mathrm{m}$ particles were observed to obtain a better focusing effect (forced into a narrower stream), which corresponds to our previous finding that the performance of particle focusing could be improved with the rise of applied electric field [41]. By setting the correction factor to be 0.4 for $15 \mu \mathrm{m}$ particles, the numerically predicted results (right column) show a good agreement with the experimentally obtained superimposed images.

\subsection{Continuous size-dependent separation of particles}

According to Eq. 2, the DEP force is proportional to the cube of particle radius, therefore, larger particles are subjected to larger DEP forces, and hence tend to be deflected further from the hurdle edge and side wall compared to smaller ones. This mechanism is utilized in the proposed microchannel to perform continuous separation of particles based on size. A typical case of the 
separation of mixed $10 \mu \mathrm{m}$ (bright) and $15 \mu \mathrm{m}$ (dark) PS particles with applied voltages at inlet A, outlet B and outlet C of 180, 0 and $18 \mathrm{~V}$, respectively, is shown in Figure 5 . The electric fields within the microchannel were numerically computed using COMSOL. The highest electric field occurred at the top edge of the hurdle in the first semi-circle is around $500 \mathrm{~V} / \mathrm{cm}$, and the average value across the entire microchannel is around $130 \mathrm{~V} / \mathrm{cm}$. Initially, the particle mixture was introduced covering nearly the whole width of inlet microchannel (see Figure 5a). Two types of particles were both focused into a single stream in the gap regions in the first curved unit, leading to a confined particle stream close to the hurdle edge before entering the second curved unit for subsequent separation (see Figure 5b). As shown in the Figure 5c, there were two distinct particle streams in the exit region, as 10 and $15 \mu \mathrm{m}$ particles were sorted and moved in a focused stream from outlet B and outlet C, respectively. The overall process was also reasonably predicted by numerical simulation (see right column of Figure 5), in which the yellow and blue lines represent the trajectories of 10 and $15 \mu \mathrm{m}$ particles, respectively, and the correction factors were fixed to be 0.5 and 0.4 , respectively, for 10 and $15 \mu \mathrm{m}$ particles. In order to test the separating efficiency, the number of two particle types (i.e. bright 10 and dark $15 \mu \mathrm{m}$ PS particles) in both outlet reservoirs were counted. Three discrete regions within each reservoir were selected for particle counting, where a total of over 300 beads were counted. The separation efficiency was calculated by $n_{r} / n_{t} \times 100 \%$ or $\left(1-n_{w} / n_{t}\right) \times 100 \%$, where $n_{t}$ is the total number of counted particles, $n_{r}$ and $n_{w}$ are the numbers of particles flowing into the right and wrong outlet, respectively. Figure 6 shows the percentages of two particle populations in the reservoirs of both outlet B and outlet C, indicating a separating efficiency of above $95 \%$.

\section{Concluding remarks}

In this work, an S-shaped microchannel embedded with multiple round hurdles for continuous manipulation and separation of microparticles using negative DC DEP was presented. In such design, the effect of obstacle and curvature are combined to induce nonuniform electric fields, which contributes to a novel technique for the continuous control of particle movement in DEP-based 
microfluidic devices. Both experimental and numerical results indicated that by adjusting applied voltages at the inlet and outlets, microparticles can be directed into distinct outlets, positioned at different locations along the outlet width direction, focused into streams of varied widths, and separated according to size.

Since the design utilized both obstacle and curvature to generate spatial nonuniformities, it provides more parameters (i.e. gap width, configuration of hurdle and curved channel, etc.) to be optimized according to the requirement of various applications, the controllability of the particle motion hence increased. More importantly, the problems that occur in the device that taking advantage of either obstacle or curvature effect individually for electric field gradients, including locally amplified electric fields, adverse effects on cell viability, particle clogging or adhesion, and large applied voltage, are greatly relieved, allowing the improvement of device performance. Furthermore, the upstream curved unit in the present design, a semi-circular microchannel combined with round hurdles, serves as a pretreatment component for driving particles into a confined stream close to the hurdle region within the downstream curved unit, which facilitates subsequent particle manipulation and separation process. It is one task of our future work that optimizing obstacle and curvature with more controllable and flexible local electric fields for new applications, such as handling, concentrating, and sorting target bioparticles (i.e. blood and cancer cells). Due to aforementioned benefits, the novel design with demonstrated integrated functionality of focusing, switching and sorting microparticles shows a great potential of using in LOC or $\mu$ TAS devices for biological, chemical, and medical applications. 


\section{Acknowledgement:}

The project was supported by the University of Wollongong through an international links project.

The author (Miss Ming Li) wishes to thank the China Scholarship Council and the University of Wollongong for offering joint PhD scholarships.

The authors have declared no conflict of interest. 


\section{References}

[1] Lim, Y. C., Kouzani, A. Z., Duan, W., Microsyst. Technol. 2010, 16, 1995-2015.

[2] Arora, A., Simone, G., Salieb-Beugelaar, G. B., Kim, J. T., Manz, A., Anal. Chem. 2010, 82, 48304847.

[3] Pohl, H. A., Dielectrophoresis: The behavior of neutral matter in nonuniform electric fields, Cambridge University Press, Cambridge 1978

[4] Lao, A. I. K., Hsing, I. M., Lab Chip 2005, 5, 687-690.

[5] Lapizco-Encinas, B. H., Ozuna-Chacon, S., Rito-Palomares, M., J. Chromatogr. A 2008, 1206, 45-51.

[6] Huang, Y., Yang, J. M., Hopkins, P. J., Kassegne, S., Tirado, M., Forster, A. H., Reese, H., Biomed. Microdevices 2003, 5, 217-225.

[7] Park, K., Akin, D., Bashir, R., Biomed. Microdevices 2007, 9, 877-883.

[8] Minerick, A. R., Zhou, R. H., Takhistov, P., Chang, H. C., Electrophoresis 2003, 24, 3703-3717.

[9] Carlson, R. H., Gabel, C. V., Chan, S. S., Austin, R. H., Brody, J. P., Winkelman, J. W., Phys. Rev. Lett. $1997,79,2149-2152$.

[10] Ramos, A., Morgan, H., Green, N. G., Castellanos, A., J. Phys. D: Appl. Phys. 1998, 31, 2338-2353.

[11] Liu, C. X., Lagae, L., Borghs, G., Appl. Phys. Lett. 2007, 90, 131102.

[12] Petersson, F., Nilsson, A., Holm, C., Jonsson, H., Laurell, T., Lab Chip 2005, 5, 20-22.

[13] Monat, C., Domachuk, P., Eggleton, B. J., Nat. Photonics 2007, 1, 106-114.

[14] Juncker, D., Schmid, H., Delamarche, E., Nature Mater. 2005, 4, 622-628.

[15] Zhang, C. X., Manz, A., Anal. Chem. 2003, 75, 5759-5766. 
[16] De A. Costa, R. C., Mogensen, K. B., Kutter, J. P., Lab Chip 2005, 5, 1310-1314.

[17] Gascoyne, P. R. C., Vykoukal, J. V., Proc. IEEE 2004, 92, 22-42.

[18] Chen, D.F., Du, H., Li, W.H., J. Micromech. Microeng. 2006, 16, 1162-1169.

[19] Zhang, C., Khoshmanesh, K., Mitchell, A., Kalantar-zadeh, K., Anal. Bioanal. Chem. 2010, 396, 401-420.

[20] Khoshmanesh, K., Nahavandi, S., Baratchi, S., Mitchell, A., Kalantar-zadeh, K., Biosens. Bioelectron. 2011, 26, 1800-1814.

[21] Gencoglu, A., Minerick, A. R., Lab Chip 2009, 9, 1866-1873.

[22] Srivastava, S. K., Gencoglu, A., Minerick, A. R., Anal. Bioanal. Chem. 2011, 399, 301-321.

[23] Shafiee, H., Caldwell, J. L., Sano, M. B., Davalos, R. V., Biomed. Microdevices 2009, 11, 997-1006.

[24] Salmanzadeh, A., Romero, L., Shafiee, H., Gallo-Villanueva, R. C. Mark A. Stremler, M. A., Cramer, S. D., Davalos, R. V., Lab Chip, 2012, 12, 182-189.

[25] Lapizco-Encinas, B. H., Simmons, B. A., Cummings, E. B., Fintschenko, Y., Anal. Chem. 2004, 76, 1571-1579.

[26] Cummings, E. B., Singh, A. K., Anal. Chem. 2003, 75, 4724-4731.

[27] Kang, K. H., Kang, Y., Xuan, X., Li, D., Electrophoresis 2006, 27, 694-702.

[28] Kang, K. H., Kang, Y., Xuan, X., Li, D., J. Appl. Phys. 2006, 99, 064702-064708.

[29] Kang, Y., Li, D., Kalams. S. A., Eid, J. E., Biomed. Microdevices 2008, 10, 243-249.

[30] Lewpiriyawong, N., Yang, C., Lam, Y. C., Biomicrofluidics 2008, 2, 034105.

[31] Hawkins, B. G., Smith, A. E., Syed, Y. A., Kirby, B. J., Anal. Chem. 2007, 79, 7291-7300. 
[32] Barbulovic-Nad, I., Xuan, X., Lee, J. S. H., Li, D., Lab Chip 2006, 6, 274-279.

[33] Thwar, P. K., Linderman, J. J., Burns, M. A., Electrophoresis 2007, 28, 4572-4581.

[34] Xuan, X., Zhu, J., Church, C., Microfluid. Nanofluid. 2010, 9, 1-16.

[35] Chen, K. P., Pacheco, J. R., Hayes, M. A., Staton, S. J. R., Electrophoresis 2009, 30, 1441-1448.

[36] Zhu, J., Tzeng, T.-R. J., Hu, G., Xuan, X., Microfluid. Nanofluid. 2009, 7, 751-756.

[37] Zhang, L., Tatar, F., Turmezei, P., Bastemeijer, J., Mollinger, J. R., Piciu, O., Bossche, A., J. Phys. $2006,34,527-532$.

[38] Chen, D., Du, H., Microfluid. Nanofluid. 2010, 9, 281-291.

[39] Zhu, J., Tzeng, T.-R. J., Xuan, X., Electrophoresis 2010, 31, 1382-1388.

[40] Zhu, J., Xuan, X., J. Colloid Interface Sci. 2009, 340, 285-290.

[41] Li, M., Li, S., Cao, W., Li, W., Wen, W., Alici, G., J. Micromech. Microeng. 2012, 22, 095001.

[42] Anderson, J. L., Annu. Rev. Fluid Mech. 1989, 21, 61-99.

[43] Ramosy, H. M. A., Greenz, N. G., Castellanosy, A., J. Phys. D: Appl. Phys. 1998, 31, 2338-2353.

[44] Jones, T. B., Electromechanics of particles, Cambridge University Press, Cambridge, 1995

[45] Takahashi, N., Aki, A., Ukai, T., Nakajima, Y., Maekawa, T., Hanajiri, T., Sens. Actuat. B: Chem. $2011,151,410-415$.

[46] Venditti, R., Xuan, X., Li, D., Microfluid. Nanofluid. 2006, 2, 493-499. 


\section{Figure legends:}

Figure 1 (a) Photograph of the DEP-based microfluidic chip for continuous manipulation and separation of microparticles. The inset indicates the structure and dimensions of the design. (b) Distribution of the electric-field lines and contours of the electric-field strength ( $\mathbf{E}$, darker region has a stronger electric field) within the microchannel. (c) Illustration of negative DEP forces (represented by dark blue arrows) exerted on particles moving through the microchannel electrokinetically.

Figure 2 Experimental (left column: snapshot; middle column: superimposed) and numerical (right column) demonstration of the effect of applied voltage at inlet $A$ on the $10 \mu \mathrm{m}$ particle motion at (a) entry, (b) centre and (c, d) exit region. The applied voltage at the inlet A was increased from (c) 140 $\mathrm{V}$ to (d) $340 \mathrm{~V}$, while outlet $\mathrm{B}$ was grounded and applied voltage at outlet $\mathrm{C}$ fixed at $15 \mathrm{~V}$ in both cases. The red arrows indicate the flow direction.

Figure 3 Experimental (left column: snapshot; middle column: superimposed) and numerical (right column) demonstration of the effect of applied voltage at outlets on the $10 \mu \mathrm{m}$ particle motion. The applied voltages at outlet B and outlet C were, respectively: (a) 15 and $0 \mathrm{~V}$; (b) 50 and $0 \mathrm{~V}$; (c) 0 and $15 \mathrm{~V}$; (d) 0 and $50 \mathrm{~V}$. In all cases, the applied voltage at inlet A was fixed at $140 \mathrm{~V}$.

Figure 4 Experimental (left column: snapshot; middle column: superimposed) and numerical (right column) demonstration of the effect of applied voltage on the $15 \mu \mathrm{m}$ particle motion. The applied voltage to inlet $A$ and outlet C were, respectively: (a) 90 and $10 \mathrm{~V}$; (b) 180 and $10 \mathrm{~V}$; (c) 180 and $30 \mathrm{~V}$. The outlet B was grounded in all cases.

Figure 5 Continuous separation of 10 and $15 \mu \mathrm{m}$ particles in S-shaped microchannel embedded with multiple round hurdles, when the applied voltages at inlet $\mathrm{A}$ and outlet $\mathrm{C}$ were 180 and $18 \mathrm{~V}$, respectively, and outlet B was grounded. Both experimental (left column: snapshot; middle column: 
superimposed) and numerical (right column) results of particle motion in the (a) entry, (b) centre and (c) exit region were presented. The red arrows indicate the flow direction.

Figure 6 Percentage of 10 and $15 \mu \mathrm{m}$ particles in the outlet reservoirs after size-dependent separation experiment in the developed microchannel.

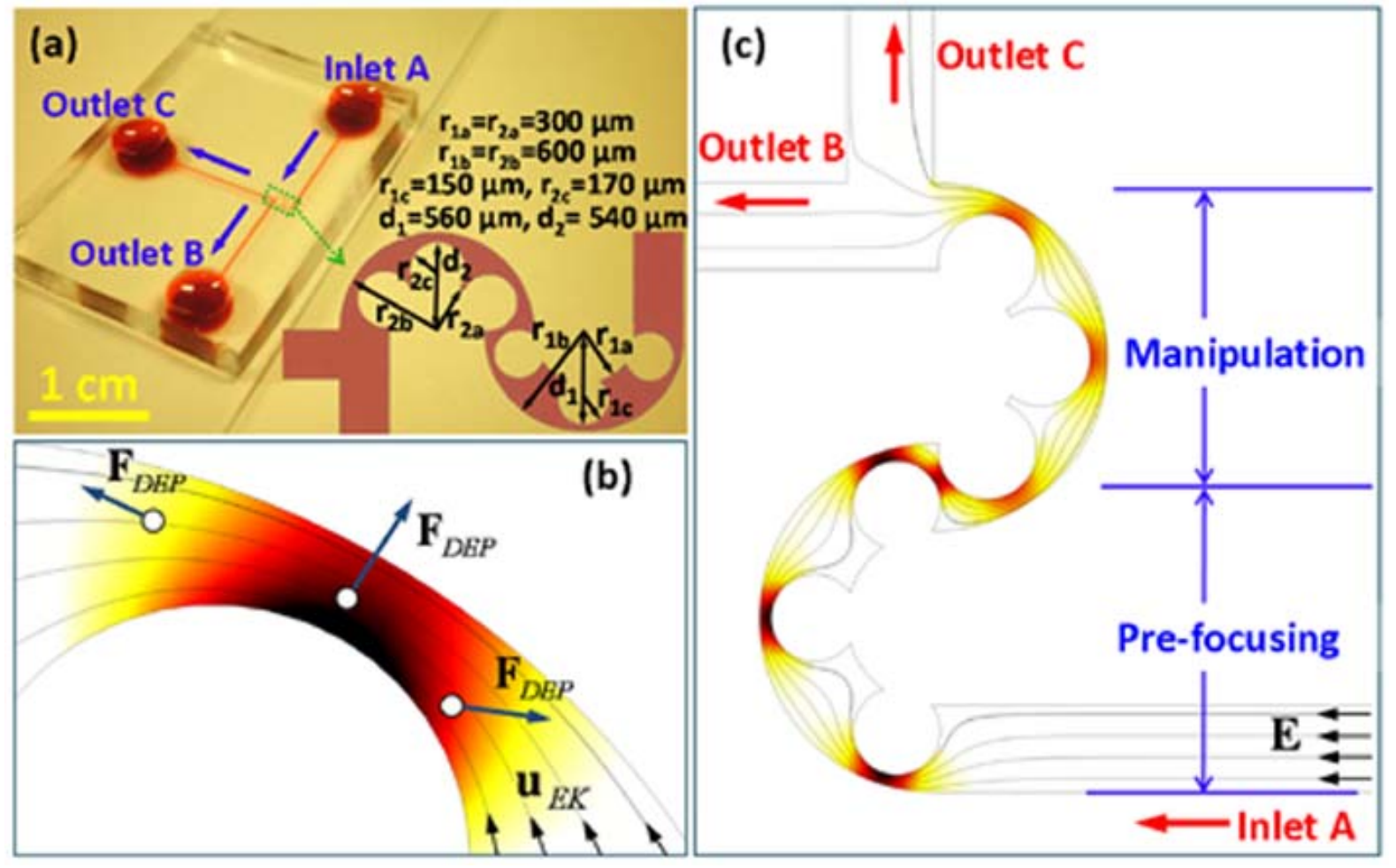

Figure 1. 


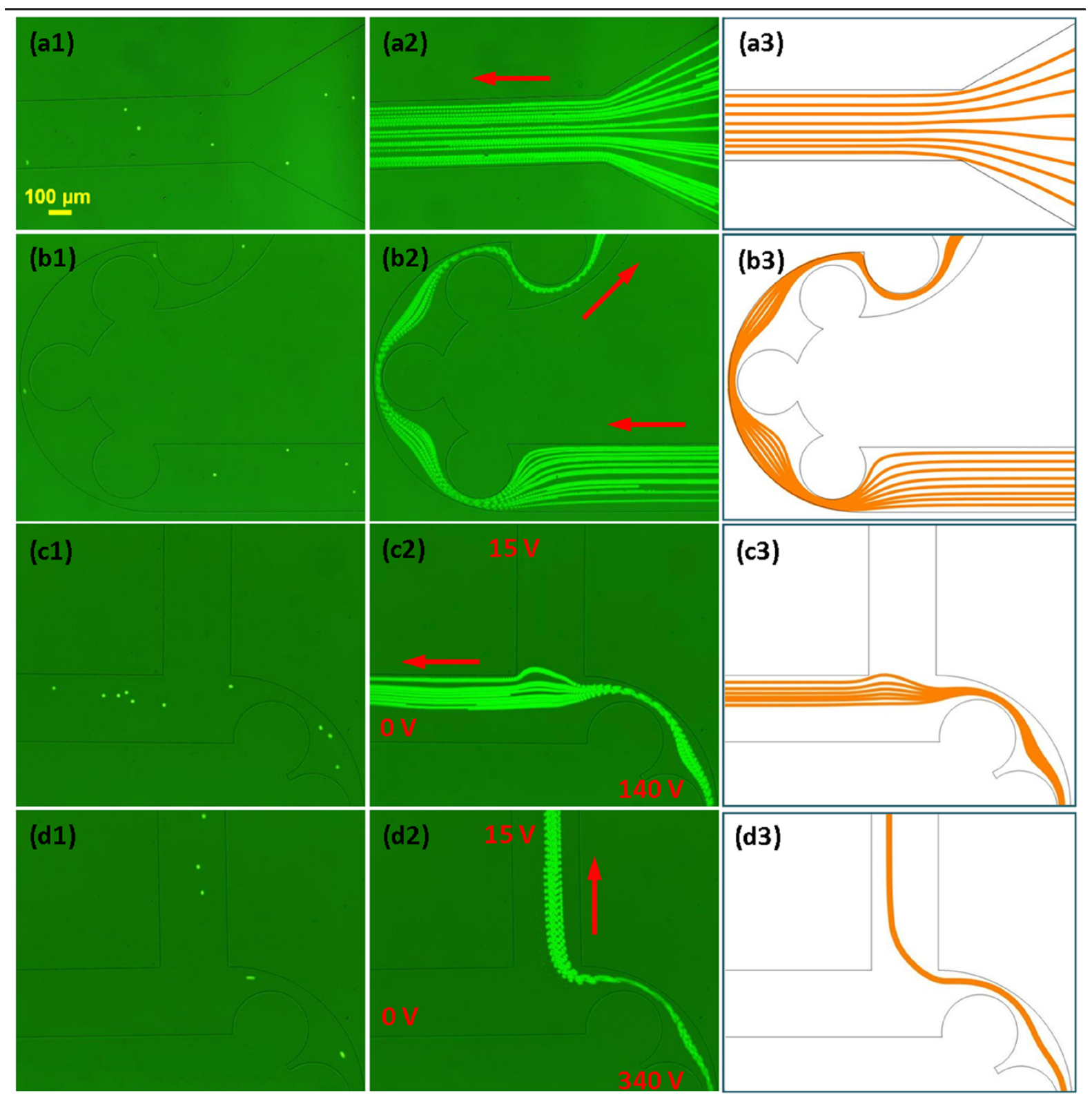

Fig. 2. 


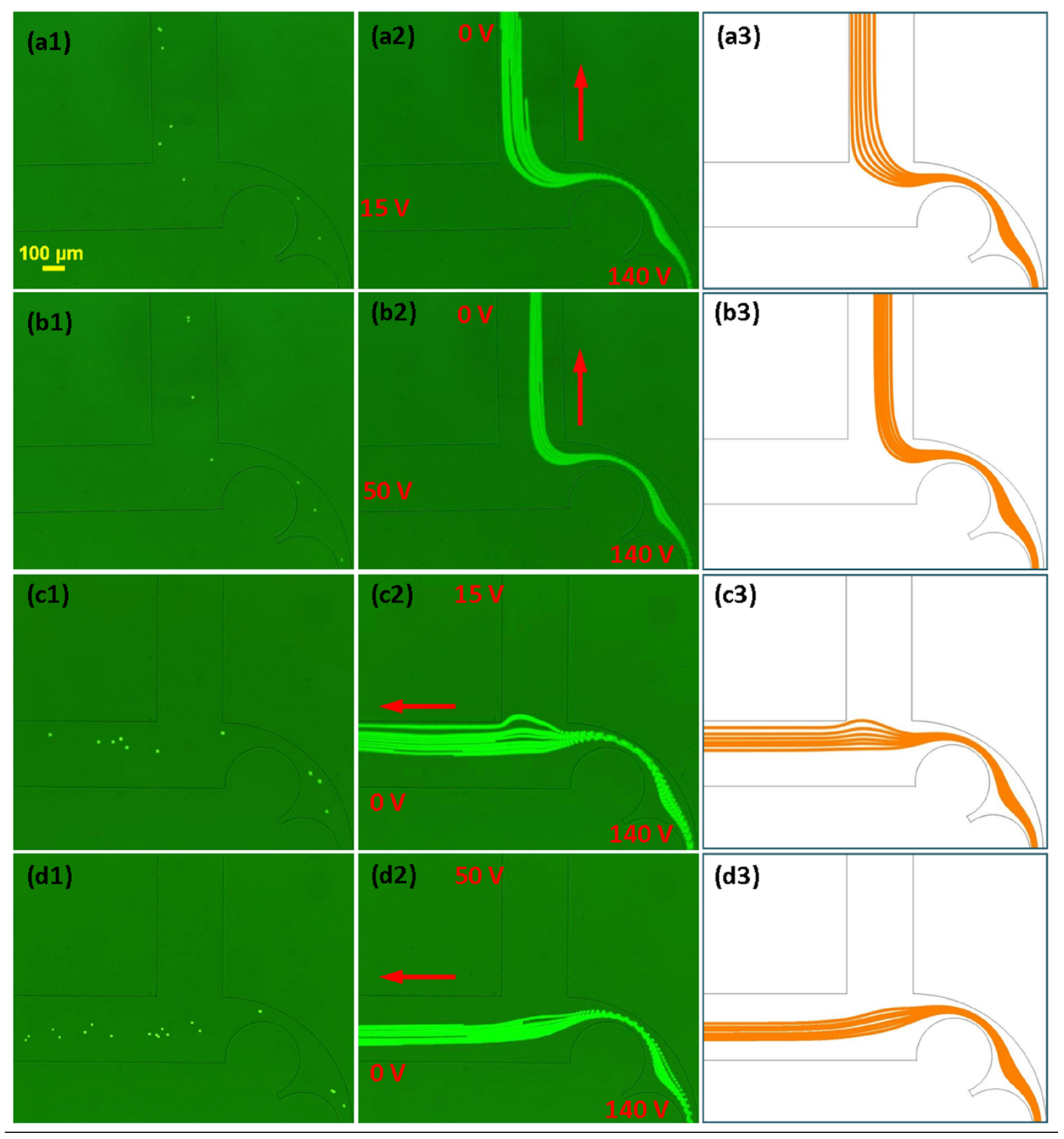

Figure 3. 


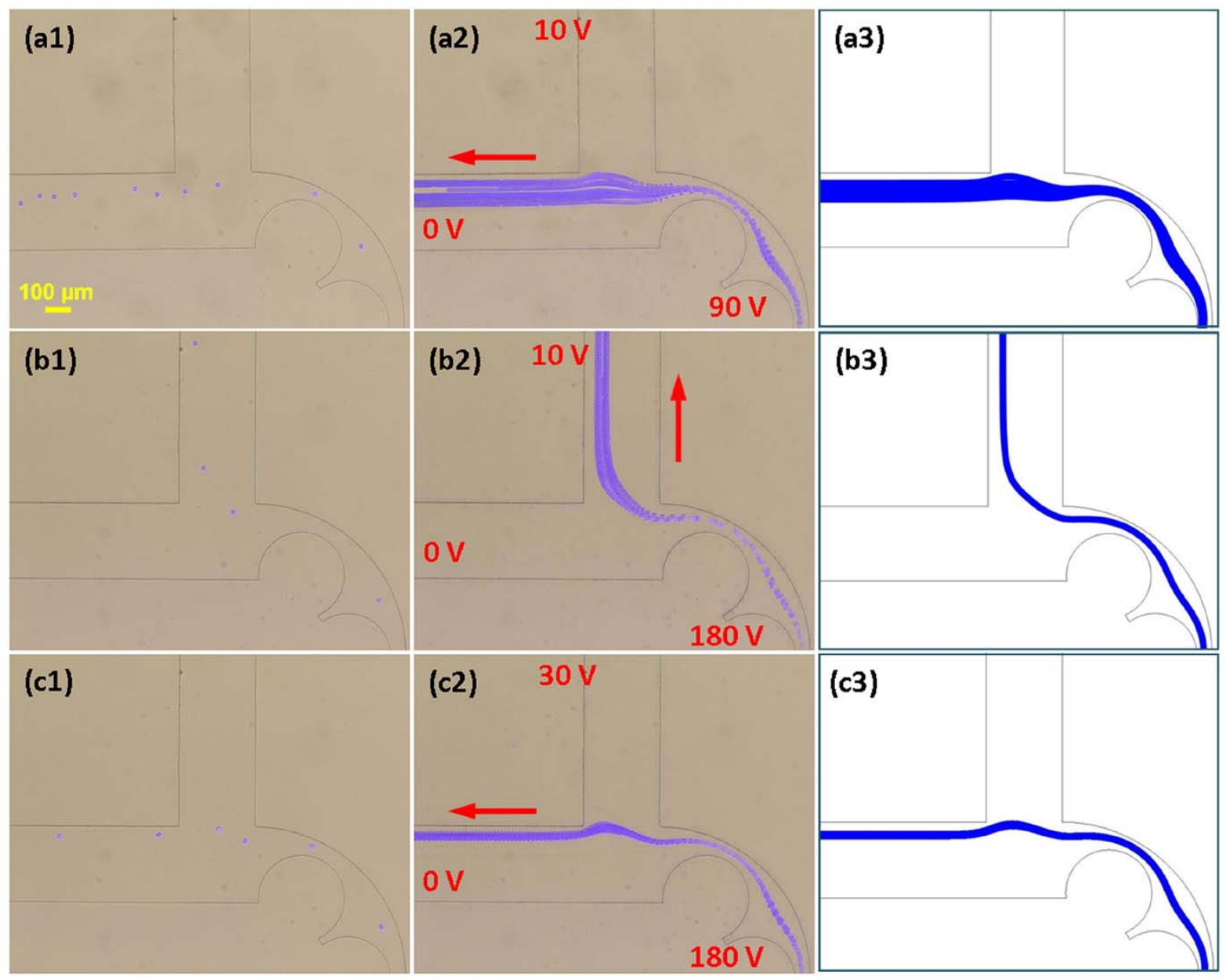

Figure 4. 


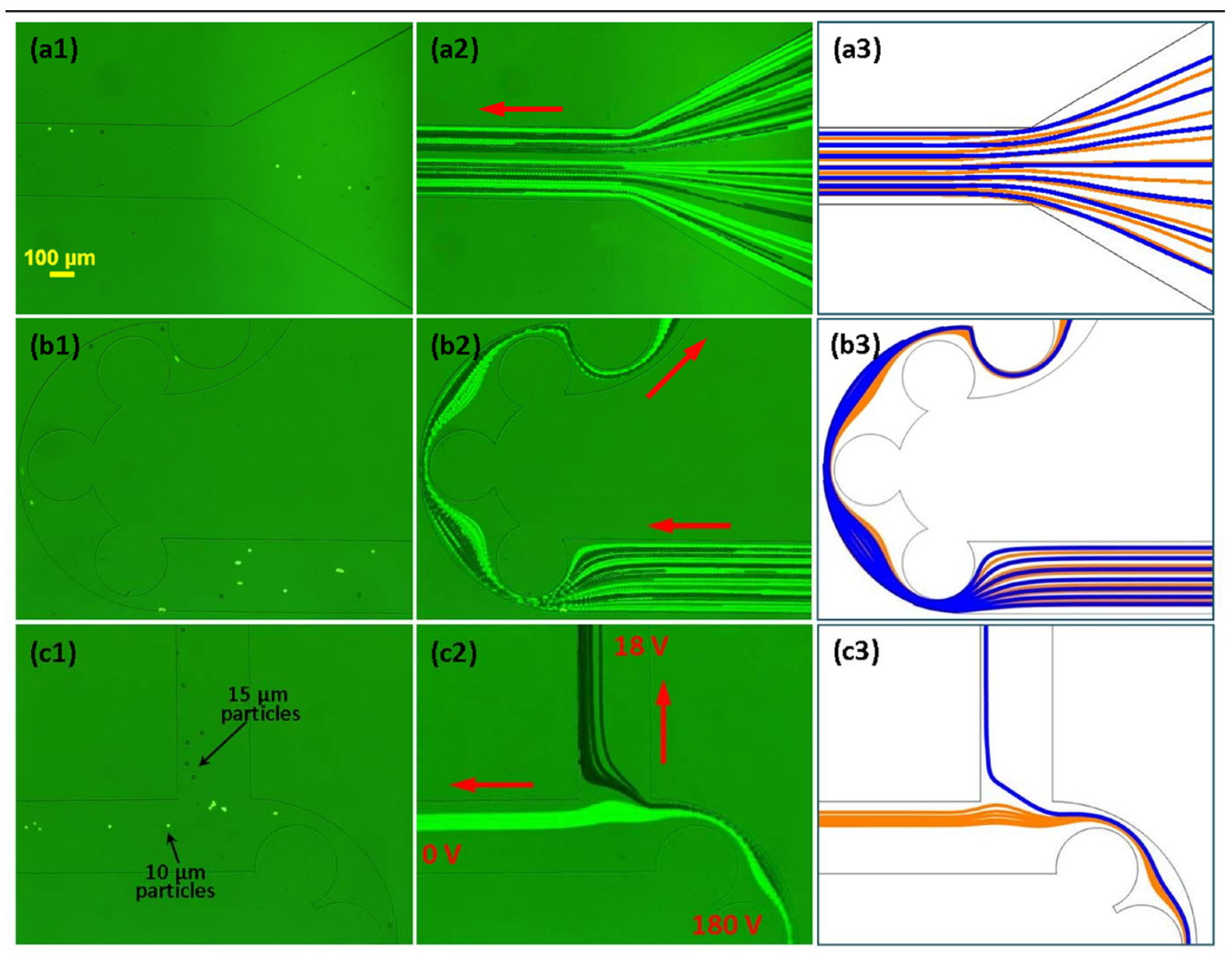

Figure 5. 


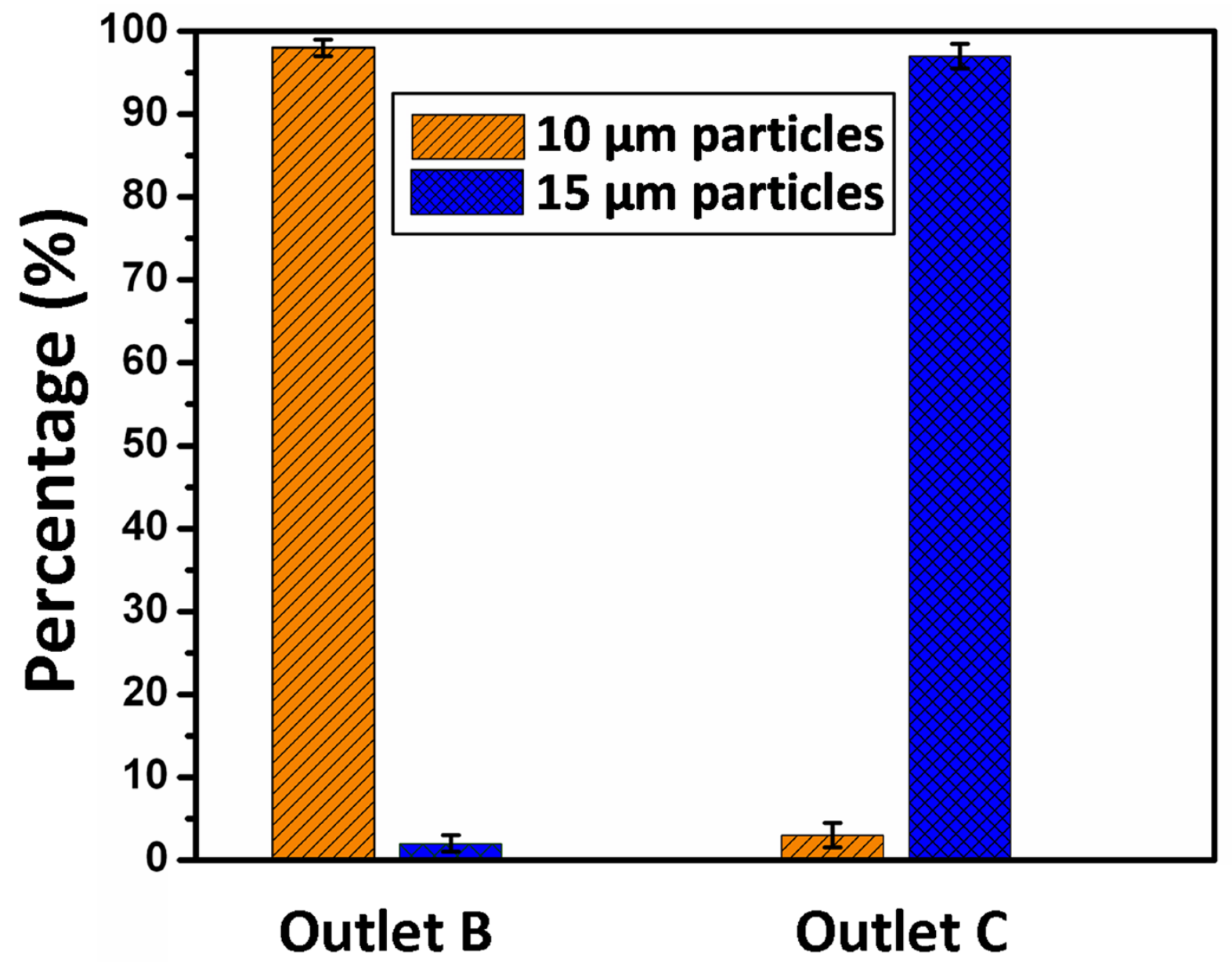

Figure 6. 